\title{
Effect of Berbamine Hydrochloride on Absorption Parameters of Berberine Hydrochloride Based on in Situ Single-Pass Intestinal Perfusion System in the Rat
}

\section{Jie Gao}

Chengdu University of Traditional Chinese Medicine Wenjiang Campus: Chengdu University of Traditional Chinese Medicine

\section{Hui Feng}

Chengdu University of Traditional Chinese Medicine Wenjiang Campus: Chengdu University of Traditional Chinese Medicine

\section{Ruohong Bi}

Chengdu University of Traditional Chinese Medicine Wenjiang Campus: Chengdu University of

Traditional Chinese Medicine

\section{Lin Pan}

Chengdu University of Traditional Chinese Medicine Wenjiang Campus: Chengdu University of

Traditional Chinese Medicine

\section{Yi Shi}

Chengdu University of Traditional Chinese Medicine Wenjiang Campus: Chengdu University of

Traditional Chinese Medicine

\section{Yunfeng Han}

Chengdu University of Traditional Chinese Medicine Wenjiang Campus: Chengdu University of Traditional Chinese Medicine

\section{Xi Tang}

Chengdu University of Traditional Chinese Medicine Wenjiang Campus: Chengdu University of Traditional Chinese Medicine

\section{Xianrong Lai ( $\nabla 105114018 @ q q . c o m$ )}

Chengdu University of Traditional Chinese Medicine Wenjiang Campus: Chengdu University of

Traditional Chinese Medicine

\section{Research}

Keywords: berberine, berbamine, synergy, P-glycoprotein, in vivo one-way intestinal perfusion

Posted Date: September 10th, 2020 
DOI: https://doi.org/10.21203/rs.3.rs-73165/v1

License: (a) (i) This work is licensed under a Creative Commons Attribution 4.0 International License. Read Full License 


\section{Abstract}

Background:Berberine is a kind of chemical that has obvious effects of lowering blood sugar, lowering blood lipid. The high toxicity of intravenous administration of berberine hydrochloride $(\mathrm{BBH})$, it is only used for oral administration in clinic,but it only about $10 \%$ bioavailability. P-glycoprotein (P-gp) efflux function affects drug bioavailability $\mathbb{B B} \mathrm{BH}$ is the substrate of $\mathrm{P}$-gp, furthermore the recognized inhibitor of P-gp (such as verapamil) can promote the absorption of $\mathrm{BBH}$. Berbamine hydrochloride(BAH) had a significant inhibitory effect on calcium influx after activation of receptor regulated calcium channels, and the calcium antagonistic effect of BAH is similar to that of verapamil.

Methods: Based on the in situ single-pass intestinal perfusion method, this article compares the absorption of the $\mathrm{BAH}$ ratio group and the $\mathrm{BBH}$ control group in different intestinal segments,and investigates their absorption parameters.

Results: The results of in situ single-pass intestinal perfusion showed that the absorption rate constant $(\mathrm{Ka})$, effective permeability coefficient (Papp), and cumulative absorption per unit area per hour $(\mathrm{Q})$ in the duodenum, jejunum and ileum of the BAH ratio group were greater than those of the BBH control group, which means that the absorption of $\mathrm{BBH}$ in the ratio group increased in various intestinal segments, and the absorption of $\mathrm{BBH}$ in the small intestine was promoted by $\mathrm{BAH}$.In addition,the mass concentration ratio of $\mathrm{BBH}$ and $\mathrm{BAH}$ group is $\mathrm{B} 40: \mathrm{A50}, \mathrm{B} 30: \mathrm{A} 20 \rrbracket$ where $\mathrm{BBH}$ is better absorbed than other groups.

Conclusions: $\mathrm{BAH}$ can indeed promote the absorption of $\mathrm{BBH}$, and improve the biological utilization of $\mathrm{BBH}$ degree. The mass concentration ratio of $\mathrm{BBH}$ and $\mathrm{BAH}$ group is $\mathrm{B} 40: \mathrm{A} 50, \mathrm{~B} 30: \mathrm{A} 20 \rrbracket$ where $\mathrm{BBH}$ is better absorbed than other groups, providing a basis for the development and research of the subsequent preparation of $\mathrm{BBH}$.

\section{Introduction}

Berberine(5,6-dihydro-9,10-dimethoxybenzo[G]-1,3-dibenzobenzo[5,6-A]quinazine) is an isoquinoline Alkaloids, whose natural sources are Berberis dictyophylla Franch, Berberis poiretii Schneid and other plants [1].The water solubility of the hydrochloride salt increased significantly. Berberine has obvious effects of lowering blood sugar, blood fat, antibacterial and anti-oxidation[2-5]. Studies have shown that $\mathrm{BBH}$ can be used to treat diabetes [6]. Because BBH intravenous administration is more toxic [7], it is only used for oral administration in the clinic. Researches have shown that[8] BBH has poor oral absorption, with only about $10 \%$ bioavailability. Increasing the bioavailability of $\mathrm{BBH}$ can better play the clinical role of $\mathrm{BBH}$, making $\mathrm{BBH}$ a new type of clinical drug for the treatment of DR.So improving the bioavailability of $\mathrm{BBH}$ is also what domestic Foreign scholars are paying attention to . In the existing research, there are two mainly two divided categories, one is to change the dosage form of $\mathrm{BBH}$ to increase its bioavailability,the second category is to add P-gp protein substrate to compete with BBH to increase its bioavailability. P-gp is often expressed in the brain, liver, kidney and small intestine, and has the function of drug efflux transport[9]. The efflux function of P-gp protein affects the bioavailability of drugs in some 
ways. Ju et al.[10]measured the effect of BBH and digoxin on the pharmacokinetics of digoxin in rats, and found that within a certain concentration range, when the two are used together, it can obviously improve the bioavailability of digoxin which is considedred to be a result of the P-gp inhibiting. Fratter et al. [9]proposed that BBH is the substrate of P-gp, and the recognized inhibitor of P-gp (such as verapamil) can promote the absorption of $\mathrm{BBH}$.

Similar to BBH, BAH (6,6',7-trimethoxy-2,2'-dimethyl-berbaman-12-o) is bis-benzyl isoquinoline alkaloids ,whose natural sources are Berberis dictyophylla Franch , Berberis poiretii Schneid and other plants[11,12] (Figure 1),berbamine has obvious effects of white blood cell proliferation, anti-inflammatory, antioxidant, anti-arrhythmic [13]. The medicinal hydrochloride is a calmodulin antagonist[14],BAH has a significant inhibitory effect on the calcium influx after receptor-mediated calcium channel activation. The calcium antagonism of BAH is similar to verapamil[14-16], under certain circumstances, it can reduce the expression of P-gp and lower its efflux function, and the antioxidant drug berbamine can prevent the DNA damage in the process of diabetic cataract to some extent [17], suggesting that BAH can be treated under certain conditions where DR also has curative effects. $\mathrm{BBH}$ is the substrate of P-gp protein. BBH has an inhibitory effect on the expression of P-gp protein, and both have therapeutic effects on DR (Figure 2). Therefore, we speculate that $\mathrm{BBH}$ can synergize with BAH for the prevention and treatment of DR.

The preliminary experiment of the research group showed that berberine can be used for the prevention and treatment of diabetes[18-22] . According to the theory of oral prevention and treatment of DR, it is concluded that the effective ingredients (berberine, berberamine, etc.) in oral berberis will be used for the treatment of DR, which Has a considerable therapeutic effect. In the theoretical system of Tibetan medicine, the "three causes theory" explains the occurrence and development of diseases (Figure 3, the balance state of "three causes", "seven essences", and "three impurities" in Tibetan medicine). Under certain conditions, Long, Chiba, and Bacon maintain coordination(coordinate) with each other and maintain the normal physiological activities of the human body. If one of them is prosperous or declining and out of balance, the balance will be destroyed, and diseases will occur. A series of complications, such as DM and DR, belong to the category of "Genny Saku's disease" (also translated as frequent urination) in Tibetan medicine.

Because BBH intravenous administration is more toxic[7], it is only used for oral administration in the clinic. The small intestine is an important organ for oral drug absorption, and intestinal absorption research can be more appropriate to draw the mechanism of drug absorption in the intestine. The oneway intestinal perfusion method[23] has the advantages of operability, better repeatability of results, and measured parameters that are closer to the actual absorption status in the body, which is more frequently used. In this paper, compared the absorption of the BAH ratio group and the BBH control group in different intestinal segments, and investigates its absorption parameters.Provide reference to explore the mechanism of $\mathrm{BBH}$ and $\mathrm{BAH}$ and $\mathrm{BBH}$ follow-up preparation study (Figure 4, the absorption route of $\mathrm{BBH}$ and $\mathrm{BBH})$.

\section{Materials And Methods}




\section{Chemicals and reagents}

BBH reference substance (Chengdu Ruifensi Biological Technology Co., Ltd., batch number: Y-035171216), BBH API (national medicine standard word: H51021627, batch number: 111101), BAH API ( National Pharmaceutical Standards: H51023712, batch number: 110402), both purchased from Chengdu Longquan High-Tech Natural Pharmaceutical Co., Ltd., acetonitrile (chromatographically pure, US Fisher), methanol (chromatographically pure, US Fisher), water is ultrapure water, two Methyl sulfoxide, sodium chloride, potassium chloride, anhydrous calcium chloride, sodium bicarbonate, potassium dihydrogen phosphate, magnesium chloride, glucose hexahydrate, chloral hydrate, physiological saline, phosphoric acid, etc. are all analytically pure.

\section{Configuration of the solution}

Krebs-Ringer (K-R) solution:Krebs-Ringer (K-R) buffer solution consisted of $7.8 \mathrm{~g} \mathrm{NaCl}, 0.35 \mathrm{~g} \mathrm{KCl}, 1.37 \mathrm{~g}$ $\mathrm{NaHCO} 3,0.22 \mathrm{~g} \mathrm{KH} 2 \mathrm{PO} 3$ and $1.40 \mathrm{~g}$ glucose dissolved in $1000 \mathrm{ml}$ of distilled water. (Note: The K-R solution is now ready for use)

The configuration of intestinal perfusate: the concentration ratio $\left(\mu \mathrm{g} \cdot \mathrm{ml}^{-1}\right)$ of $\mathrm{BBH}(\mathrm{B})$ and $\mathrm{BAH}(\mathrm{A})$ were $60: 40,50: 10,40: 50,30: 20,20: 60,10: 30$. Precisely weigh the appropriate amount of BBH and BAH, add KR solution containing 1\% dimethyl sulfoxide, and obtain the above 6 groups of intestinal perfusion with different concentrations. In addition, accurately weigh the appropriate amount of BBH and add K-R solution containing $1 \%$ of dimethyl sulfoxide to obtain high, medium and low concentrations of 60.47 , 30.16 and $10.02 \mu \mathrm{g} \cdot \mathrm{ml}^{-1}$ of BBH intestinal perfusate.

The configuration of the reference solution: $\mathrm{BBH}$ was dissolved in $\mathrm{K}-\mathrm{R}$ buffer solution to make drug concentrations set at $0.25,0.5,1,2,4$, and $8 \mu \mathrm{g} \cdot \mathrm{ml}^{-1}$.

Configuration of chloral hydrate: Precisely weigh $500 \mathrm{mg}$ of chloral hydrate, dissolve it with $0.9 \%$ physiological saline, and dilute to $50 \mathrm{ml}$ volumetric flask to obtain $10 \%$ chloral hydrate solution. The amount of injection for rats is about $0.35 \mathrm{ml}$ of $10 \%$ chloral hydrate solution per $100 \mathrm{~g}$.

\section{Handling of samples}

$0.5 \mathrm{ml}$ of perfused intestinal fluid was measured, methanol of $0.5 \mathrm{ml}$ was added and swirled for 3 minutes. The supernatant was centrifuged for 10 minutes at the rotational speed of $11000 \mathrm{r} \cdot \mathrm{min}^{-1}$. The supernatant was filtered through $0.22 \mu \mathrm{m}$ microporous membrane, and the filtrate was prepared.

\section{Determination of index components in intestinal irrigation by high performance liquid chromatography}

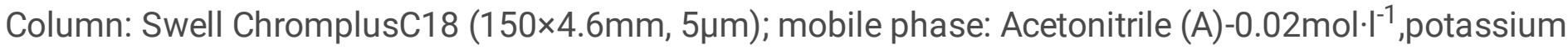
dihydrogen phosphate $(\mathrm{B}, \mathrm{pH}=2.8)$, gradient elution $(0 \sim 35 \mathrm{~min}, 22 \% \sim 28 \% \mathrm{~A})$; column temperature: $30^{\circ} \mathrm{C}$; detection wavelength: $270 \mathrm{~nm}$; flow rate: $0.6 \mathrm{~mL} \cdot \mathrm{min}-1$; injection volume: $10 \mu \mathrm{l}$. And the investigation of 
specificity, preparation of standard curve, blank recovery rate experiment, precision experiment, stability test, physical adsorption of BBH solution in rat intestinal wall were investigated.

\section{Animals and experimental processes [23]}

SPF grade SD male rats (Chengdu Dashuo Experimental Animal Breeding Co., Ltd., certificate number: SCXK (Chuan) 2015-030), body weight $(220 \pm 20) \mathrm{g}$, healthy and qualified, ready for use after quarantine.

$0.9 \%$ normal saline, intestinal perfusion solution, Eppendorf tube (hereinafter referred to as EP tube) filled with perfusion solution are placed in a $37 \pm 0.5^{\circ} \mathrm{C}$ constant temperature water bath for preheating and keeping warm.Animals were divided into different experimental groups and fasted for $18 \mathrm{~h}$ with waterad libitum before the experiment.Anesthetized with $10 \%$ chloral hydrate (i.p.; $3.5 \mathrm{ml} \cdot \mathrm{kg}^{-1}$ ), the rats were fastened to a plate to maintain a temperature of $37 \pm 0.5^{\circ} \mathrm{C}$.

Each intestine segment was cut at both ends of about $10-15 \mathrm{~cm}$ in length ,silicone tubing was inserted and tied tightly with surgical thread. The wound was covered with degreased gauze soaked in saline to keep it moist, and the temperature was maintained with a light lamp.The micro peristaltic pump uses $5 \mathrm{ml} \cdot \mathrm{min}-1$ to pump $37 \pm 0.5^{\circ} \mathrm{C}$ normal saline to clean the intestines, and then drains the normal saline in the intestines and pipes, and then an appropriate amount of intestinal perfusion solution containing reagents is added to $1 \mathrm{ml} \cdot \mathrm{min}^{-1}$ is pumped into the intestine at a fast flow rate. The experimental process of the in vivo unidirectional intestinal perfusion method is shown in Figure 5 (Figure 5). At the end of the experiment, the rats were killed with an overdose of anesthetic, and the perfused intestinal segment was quickly cut, and its length $(\mathrm{L})$ and cross-sectional radius $(r)$ were measured. Selection of different bowel segments: the duodenum segment is about $1 \mathrm{~cm}$ down from the pylorus and $10 \mathrm{~cm}$ down; the jejunum segment is about $15 \mathrm{~cm}$ down from the pylorus and down $10 \mathrm{~cm}$; the ileum segment is about $20 \mathrm{~cm}$ down from the cecum and $10 \mathrm{~cm}$ down; the whole bowel segment is from The pylorus is about $1 \mathrm{~cm}$ down to $10 \mathrm{~cm}$ up the cecum.

\section{The effect of different $\mathrm{pH}$ of perfusate on the absorption of whole intestine of $\mathrm{BBH}$ and $\mathrm{BAH}$}

The concentration ratio of $\mathrm{BBH}$ and $\mathrm{BAH}$ were selected as 30:20 perfusate to optimize the optimal $\mathrm{pH}$ for rat intestinal absorption. The $\mathrm{NaOH}$ solution of $0.1 \mathrm{~mol} \cdot \mathrm{I}^{-1}$ was used to adjust the $\mathrm{pH}$ to $6.0,7.4,8.0$, and the blank K-R solution with different $\mathrm{pH}$ was obtained. The appropriate amount of $\mathrm{BBH}$ and $\mathrm{BAH}$ were dissolved in blank perfusion solution of different $\mathrm{pH}$ to make the concentration ratio of $\mathrm{BBH}$ and $\mathrm{BAH}$ at 30:20. The experiment was carried out according to the "2.5" method. After the sample was disposed of under "2.3", the chromatographic determination was carried out according to "2.4", and the peak area was recorded and calculated. The absorption of berberine hydrochloride and berbamine hydrochloride at 30:20 in different $\mathrm{pH}$ conditions was investigated.

\section{The effect of different concentrations of BBH on the absorption of various intestinal segments}


According to the results of "2.6" experiment, K-R solution containing 1\% dimethyl sulfoxide (DMSO) with $\mathrm{pH} 7.4$ was used as solvent to prepare intestinal perfusion solution with different concentrations, the high, medium and low concentrations of BBH were $60.47,30.16$ and $10.02 \mu \mathrm{g} \cdot \mathrm{ml}^{-1}$. The duodenum, jejunum and ileum of rat small intestine were perfused following the method of "2.5",the samples were disposed under "2.3", and the chromatographic determination was carried out following "2.4". The effects of different concentrations of $\mathrm{BBH}$ on intestinal absorption in rats were investigated.

\section{The effect of different concentrations of BBH and BAH on the absorption of various intestinal segments}

According to the results of "2.6" experiment, K-R solution containing $1 \%$ dimethyl sulfoxide (DMSO) at pH 7.4 was selected as the solvent to explore the effect of different concentrations of BAH on BBH.

According to the uniform design method, the ratio of the concentration $\left(\mu \mathrm{g} \cdot \mathrm{ml}^{-1}\right)$ of $\mathrm{BBH}(\mathrm{B})$ and $\mathrm{BAH}(\mathrm{A})$ with 4 factors and 6 levels is designed to be 60:40, 50:10, 40:50, 30:20, 20:60, 10:30, with different ratios of intestinal perfusion solution. Following method of " 2.5 ", the perfusion experiment of duodenum, jejunum and ileum of rat small intestine was carried out.,the samples were disposed referring to " 2.3 " and determined by chromatography referring to "2.4". The effects of different concentrations of BBH and BAH on intestinal absorption in rats were investigated.

\section{Calculation of experimental parameters of in situ intestinal perfusion in rats}

In this test, the volume of the inflow and outflow of the perfusion solution is corrected by the weight method to eliminate the influence of the volume change. Accurately measure $0.5 \mathrm{~mL}$ of perfusion fluid into an EP tube of known mass, weigh the total mass of the liquid and EP tube, and calculate the density $\rho$, assume that the density does not change before and after perfusion. Use the following formula to calculate the drug absorption rate constant (Ka), effective permeability coefficient (Papp) and cumulative absorption per unit area per hour (Q). In the formula, Vin and Vout are the volume of perfusion fluid infused and collected, Cin and Cout are the drug concentration in the perfusion fluid in and out of the inlet, $L$ and $r$ are the length and radius of the intestine segment under investigation, $u$ is the perfusion velocity. 


$$
\begin{aligned}
& K a=\left(1 \frac{\mathrm{C}_{\text {out }} \mathrm{V}_{\text {out }}}{\mathrm{C}_{\text {in }} \mathrm{V}_{\text {in }}}\right) \times \frac{\mathrm{u}}{\pi \mathrm{r}^{2} \mathrm{~L}} \quad \text { (Formula 1) } \\
& P a p p=\frac{-\mathrm{uln}\left(\frac{\mathrm{C}_{\text {out }} \mathrm{V}_{\text {out }}}{\mathrm{C}_{\text {in }} \mathrm{V}_{\text {in }}}\right)}{2 \pi \mathrm{rL}} \quad \text { (Formula 2) } \\
& Q=\frac{\mathrm{C}_{\text {in }} \mathrm{V}_{\text {in }}-\mathrm{C}_{\text {out }} \mathrm{V}_{\text {out }}}{2 \pi \mathrm{rL}} \quad \text { (Formula 3) }
\end{aligned}
$$

\section{Statistical methods}

The results were analyzed by SPSS21.0 software, and the results were expressed as $\pm s$, the difference between groups was analyzed by one-way (ANOVA). The absorption rate constant Ka of BBH and BBH and $\mathrm{BAH}$ in different concentrations, different $\mathrm{pH}$ and different intestinal segments were significantly different $(P<0.05)$.

\section{Results}

\section{$\mathrm{HPLC}$ analysis of $\mathrm{BBH}$ and $\mathrm{BAH}$ in the intestinal perfusion solution}

Specificity investigation (Fig. 6) it is known that the peak time of berbamine hydrochloride and berberine hydrochloride is about $7.2 \mathrm{~min}$ and $26.5 \mathrm{~min}$, the peak shape of the index peak is good, and the retention time of the index components in the sample is basically the same as that in the control sample, indicating that the chromatographic condition has a good specificity, and the mass range of good linear relationship between berberine hydrochloride and peak area is $2.5 \sim 80 \mu \mathrm{g}$. The blank recovery experiment showed that the blank recovery rates of high, medium and low concentration of berberine hydrochloride were $98.77 \%, 95.17 \%$ and $95.49 \%$, respectively, indicating that the blank recovery rate met the requirements. The precision test results showed that the range of RSD of inter-day precision and intraday precision of high, medium and low concentration of berberine hydrochloride was $0.33 \%$ and $0.88 \%$, indicating that the precision of the instrument was good. The stability test results show that the RSD value is $0.97 \%$, indicating that the test stability is good.

Investigation results of the physical adsorption of $\mathrm{BBH}$ solution on the intestinal wall of rats: SD male rats fasted for 18 hours were taken, and each intestine segment of the rat small intestine was quickly cut open after anesthesia, and then killed by excessive anesthesia. In $0.9 \%$ saline, the intestine segment was carefully peeled off the surface tissue, and then the mucosa was carefully turned out with a self-made silicone rod, rinsed with $0.9 \%$ saline, and placed in the intestinal perfusion solution (the concentration of 
$\mathrm{BBH}$ is $30.16 \mu \mathrm{g} \cdot \mathrm{ml}^{-1}$ ), incubate in a water bath at $37 \pm 0.5^{\circ} \mathrm{C}$ for $3 \mathrm{~h}$, remove the intestine segment, determine the content of $\mathrm{BBH}$ in the intestinal perfusate after incubation, calculate the residual rate after incubation, duodenum, jejunum, The RSD of the ileum was $1.70 \%, 1.91 \%$, and $1.46 \%$. The results showed that there was no obvious physical adsorption effect of $\mathrm{BBH}$ on the small intestine wall of rats.

\section{The effect of perfusate with different pH on the absorption of whole intestine of BBH and BAH (Fig. 7)}

When the concentration ratio of $\mathrm{BBH}$ and $\mathrm{BAH}$ was $\mathrm{B} 30: \mathrm{A} 20$, at $\mathrm{pH} 6.0,7.4,8.0$, after analysis of variance,the $\mathrm{Ka}, \mathrm{Papp}$ of $\mathrm{BBH}$ in $\mathrm{pH} 7.4$ and 8 perfusate was significantly different from that in $\mathrm{pH} 6(\mathrm{PV}$ 0.05), but there was no significant difference between $\mathrm{pH} 7.4$ and $\mathrm{pH} 8$. As shown in figure 7, the Papp and $\mathrm{Ka}$ of $\mathrm{BBH}$ in the perfusion solution of $\mathrm{pH} 7.4$ are higher than those of $\mathrm{pH} 6$ and $\mathrm{pH} 8$, indicating that the intestinal absorption of $\mathrm{BBH}$ is relatively good in neutral environment. Therefore, the perfusion solution $\mathrm{pH}$ of this experiment is 7.4 .

\section{The effect of high, medium and low concentrations of BBH on the absorption of various intestinal segments}

The absorption parameters of different mass concentrations of $\mathrm{BBH}$ in each segment of the intestine (Figure 8),according to one-way analysis of variance, when the mass concentration of BBH perfusion solution was $10 \sim 60 \mu \mathrm{g} \cdot \mathrm{ml}^{-1}$, there was no significant difference in $\mathrm{Ka}$ among the three different mass concentrations in duodenum, jejunum and ileum. The cumulative absorption of drugs in the duodenum, jejunum, and ileum increased linearly with the increase of the drug concentration of the perfusate, showing that there does not exist saturated absorption of $\mathrm{BBH}$ in the mass concentration of $10 \sim 60 \mu \mathrm{g} \cdot \mathrm{ml}^{-}$

1 . The absorption of berberine in the duodenum, jejunum, and ileum may be passive diffusion, which is in line with the first-order process of absorption kinetics.After one-way analysis of variance, when the mass concentration of $\mathrm{BBH}$ perfusate was $10 \sim 60 \mu \mathrm{g} \cdot \mathrm{ml}^{-1}$, the Ka of duodenum was significantly different from the $\mathrm{Ka}$ of jejunum and ileum at the same concentration( $\left.{ }^{\star} \mathrm{P} \otimes 0.05\right)$, the concentration of jejunum $\mathrm{Ka}$ and ileum Ka also contained a significant difference $\left({ }^{\#} \mathrm{P}<0.05\right)$. As shown in figure 8 , the Papp and Q of jejunum were significantly higher than those of jejunum and ileum at the same concentration. The results showed that $\mathrm{BBH}$ was absorbed by the whole intestine, and the absorption of $\mathrm{BBH}$ was different in different intestinal segments, and its absorption decreased in the order of jejunum, duodenum and ileum.

\section{The effect of different concentrations of BBH and BAH on the absorption of each intestinal segment (Fig.9, Fig.10)}

Figure 9A, 9B, 9C BBH and $\mathrm{BAH}$ different concentration ratio group in each intestine absorption parameters.Figure 10A, 10B, 10C Q-t curves of different ratios of $\mathrm{BBH}$ and $\mathrm{BAH}$ absorbed in each intestinal segment.Through analysis of variance, the Ka of B40:A50 and B30:A20 in duodenum, jejunum and ileum in $\mathrm{BBH}$ and $\mathrm{BAH}$ group were significantly different from those in other groups in the same intestinal segment, but there was no significant difference in B40:A50 and B30:A20, and there was no significant difference in Papp and Q in B40:A50 and B30:A20 between other groups in the same intestinal 
segment and other matching groups in the same intestinal segment, and the Papp and Q of berberine and berbamine in duodenum, jejunum and ileum were also higher than those in other groups in the same intestinal segment. The results showed that when the mass concentration of BBH was $10 \sim 60 \mu \mathrm{g} \cdot \mathrm{ml}^{-1}$, the mass concentration ratio of $\mathrm{BBH}$ to $\mathrm{BAH}$ was $\mathrm{B} 40: \mathrm{A} 50$ and $\mathrm{B} 30: \mathrm{A} 20$, which was better than that of other groups.

From Figures $8 \mathrm{~A}, 8 \mathrm{~B}, 8 \mathrm{C}$ and Figures $9 \mathrm{~A}, 9 \mathrm{~B}, 9 \mathrm{C}$, the $\mathrm{BBH}$ and $\mathrm{BAH}$ ratio group and the $\mathrm{BBH}$ alone group are compared with the same intestinal segment of the same BBH mass concentration. The Ka, Papp, and $\mathrm{Q}$ in the duodenum, jejunum, and ileum of the ratio group were greater than those of the BBH group alone, indicating that the absorption of $\mathrm{BBH}$ in the ratio group increased to varying degrees in each intestinal segment. BAH can promote the absorption of $\mathrm{BBH}$ in the small intestine, which may be related to the inhibitory effect of P-gp protein,those may have a synergistic effect.

\section{Discussion}

From the previous experiment, it was found that the Papp and Ka of berberine hydrochloride in the perfusion solution of $\mathrm{pH} 7.4$ were higher than those of $\mathrm{pH} 6$ and $\mathrm{pH} 8$, so the $\mathrm{pH}$ of the perfusion solution in this experiment was 7.4. The absorption of $\mathrm{BBH}$ in the small intestine decreased in the order of jejunum, duodenum and ileum. Compared with the $\mathrm{BBH}$ control group, the $\mathrm{BBH}$ in the $\mathrm{BBH}$ and $\mathrm{BAH}$ group was larger than the Ka, Papp and Q in the control group, the results showed that the absorption of $\mathrm{BBH}$ in each intestinal segment in the matching group increased in varying degrees,and indicating that BAH promoted the absorption of $\mathrm{BBH}$ in the small intestine, which may be related to the inhibition of P-gp protein. The Ka with mass concentration ratios of $\mathrm{B} 40: \mathrm{A} 50$ and $\mathrm{B} 30: \mathrm{A} 20$ in the proportioning group is significantly different from other proportioning groups,the results showed that when the mass concentration of $\mathrm{BBH}$ was $10 \sim 60 \mu \mathrm{g} \cdot \mathrm{ml}^{-1}$, the mass concentration ratio of $\mathrm{BBH}$ to $\mathrm{BAH}$ was $\mathrm{B} 40: \mathrm{A} 50$ and $\mathrm{B} 30: \mathrm{A} 20$, which was better than that of other groups.

In the process of absorption in the small intestine, both the drug and water are absorbed, so that the volume of the perfusate is reduced where the remaining drug amount cannot be directly calculated. In the early stage of the experiment, the change of the volume of the perfusate calibrated by the phenol red method was also investigated, but because of its cumbersome experimental steps and limited laboratory conditions, and the phenol red itself can be absorbed[24], it interferes with the intestinal transport or analytical determination of some compounds, and then uses the gravimetric method to correct the change of perfusion volume before and after perfusion, so as to ensure that the liquid volume basically does not change.The gravimetric method is also more suitable for the study of poorly soluble and difficult to absorb drug absorption mechanism. Compared with the phenol red method, it only needs to determine the concentration of the drug in the perfusate, and does not involve the determination of phenol red, which makes the experimental procedure more concise and reduces the detection workload[24]. The insufficiency of the gravimetric method assumes that the density of the inlet and outlet perfusate remains constant, but various reasons such as absorption and secretion of water by the intestine, peeling of 
mucous membranes, and absorption of drugs can cause the density to change, which is contrary to the setting,that will bring cause errors[25].

There are many factors affecting the intestinal absorption of drugs, but the dissolution of drugs in intestinal fluid and intestinal mucosa is an important prerequisite for drug absorption. Due to the incomplete dissolution of berberine hydrochloride in Kmurr solution, the use of $1 \%$ dimethyl sulfoxide was determined by reading the literature [26] and previous investigation. The bioavailability of the drug is affected by $\mathrm{pH}$, and the $\mathrm{pH}$ value of the drug is closely related to the absorption of the drug in the intestinal tract. In this paper, three $\mathrm{pH}$ values were selected and the parameters of intestinal absorption were calculated by consulting the literature [27-29] and pre-experiment, the results showed that when the $\mathrm{pH}$ was 7.4,8, there was a significant difference between $\mathrm{BBH} \mathrm{Ka}$ and $\mathrm{pH} 6$, and the $\mathrm{Ka}$ and $\mathrm{Papp}$ of $\mathrm{pH} 7.4$ were higher than $\mathrm{pH} 8$, so the perfusion solution $\mathrm{pH}$ was 7.4.

The results of this experiment show that when $\mathrm{BBH}$ is in the mass concentration range of $10 \sim 60 \mu \mathrm{g} \cdot \mathrm{ml}^{-1}$, the Ka, Papp and Q of $\mathrm{BBH}$ in the matching group are greater than that of the $\mathrm{BBH}$ control group alone,the absorption of B40: A50 and B30: A20 in the BAH group was better than that in the small intestine of rats, indicating that $\mathrm{BAH}$ can indeed promote the absorption of $\mathrm{BBH}$,and improve the biological utilization of $\mathrm{BBH}$ degree, this may be related to P-gp transport efflux. P-gp is a substrate drug that can be discharged from the intestinal epithelial cells back into the intestine, which has an effect on the absorption of the drug in the intestine. It has the specificity of substrates, inhibitors and inducers. Transporter inhibitors and their substrates Simultaneous use can cause competitive or non-competitive interactions with transporters at the molecular level [30]. The physical and chemical characteristics of the drug, the structure of the intestinal wall, and the intestinal enzymes, intestinal epithelial cells, etc. on drug absorption and metabolism all determine whether the drug can be absorbed orally[30]. The transport membrane protein P-gp in the epithelial cells of the small intestine can drain the substrate drug from the intestinal epithelial cells back to the intestine, which will affect the absorption of the drug in the intestine, so the efflux of P-gp protein is certain to a certain extent affects the bioavailability of drugs. It has been proved in the literature that $\mathrm{BBH}$ is a substrate drug of P-gp, and the inhibitor of P-gp can promote the absorption of $\mathrm{BBH}[9,31], \mathrm{BAH}$ is a calcium antagonist,the P-gp inhibitor verapamil is the same[14-16,11], which can inhibit the expression of $\mathrm{P}$-gp, suggesting that $\mathrm{BAH}$ can synergistically cooperate with $\mathrm{BBH}$ to improve the biological activity of BBH Utilization. In future experiments, we can compare the ratio of $\mathrm{BAH}$ and common P-gp inhibitors (such as verapamil) with $\mathrm{BBH}$ to intestinal absorption and determine which inhibitor can increase BBH. The absorption of alkali, and to explore oral materials to promote the absorption and transport of $\mathrm{BBH}$, providing a basis for the development and research of the subsequent preparation of $\mathrm{BBH}$.

\section{Conclusion}

In this paper, the ratio of $\mathrm{BBH}$ to $\mathrm{BAH}$ based on intestinal absorption was studied, the results showed that $\mathrm{BBH}$ and $\mathrm{BAH}$ had a certain synergistic effect in intestinal absorption.It is also concluded that the Ka with mass concentration ratios of B40:A50 and B30:A20 in the proportioning group is significantly different 
from other proportioning groups,the results showed that when the mass concentration of $\mathrm{BBH}$ was $10 \sim 60 \mu \mathrm{g} \cdot \mathrm{ml}-1$, the mass concentration ratio of $\mathrm{BBH}$ to $\mathrm{BAH}$ was $\mathrm{B} 40: \mathrm{A} 50$ and $\mathrm{B} 30: \mathrm{A} 20$, which was better than that of other groups. This paper provides a basis for the improvement of the follow-up bioavailability of $\mathrm{BBH}$, and $\mathrm{BBH}$ has the effects of reducing blood sugar and blood lipids, on the one hand, $\mathrm{BAH}$ has a certain effect on the treatment of diabetic cataract, on the other hand, BAH can increase the bioavailability of $\mathrm{BBH}$, the ratio of the two is expected to become a clinical drug for the prevention and treatment of DR in the future.

\section{Abbreviations}

BBH:berberine hydrochloride;BAH:berbamine hydrochloride; P-gp: P-glycoprotein ; Ka:the absorption rate constant;Papp:effective permeability coefficient ;Q: cumulative absorption per unit area per hour.

\section{Declarations}

\section{Acknowledgements}

Not applicable.

\section{Funding}

This research was funded by the National Natural Science Foundation of China (no.81774007,81473427)

\section{Authors' contributions}

Jie Gao: wrote the paper; Xianrong La,Hui Feng:conceived and designed the study; andLin Pan, Ruohong Bi , Yi Shi , Yunfeng Han, Xi Tang: amended the paper.

\section{Availability of data and materials}

All data generated or analysed during this study are included in this published article and its supplementary information fles.

\section{Ethics approval and consent to participate}

Not applicable.

\section{Consent for publication}

Not applicable.

\section{Competing interests}

The authors declare that they have no competing interests. 


\section{References}

1.Neag M, Mocan A, Echeverría J ,Pop RM,Bocsan Cl,Crişan G,et al. Berberine: Botanical Occurrence, Traditional Uses, Extraction Methods, and Relevance in Cardiovascular, Metabolic, Hepatic, and Renal Disorders.Frontiers in pharmacology .2018;9:557.

2.Belwal T, Bisht A, Devkota HP, Ullah H, Khan H, Pandey A and,et al.

Echeverría,BerberisPhytopharmacology and Clinical Updates of Species Against Diabetes and Other Metabolic Diseases. Frontiers in pharmacology .2020;11: 41.

3.Hu YS, Gareth E, Davies.Berberine inhibits adipogenesis in high-fat diet-induced obesity mice.Fitoterapia . 2010;81( 5 ) :358-366.

4.Yong Y, Li YX, Gang LX, Jing Z, Bao SZ,Lu Y.Synthesis and Antimicrobial Activity of 8-Alkylberberine Derivatives with a Long Aliphatic Chain.Planta Med .2007;73:602-604.

5.Kaboli PJ, Rahmat A,Ismail P,Ling KH. Targets and mechanisms of berberine, a natural drug with potential to treat cancer with special focus on breast cancer. European journal of pharmacology .2014;740:584-95.

6.Chang WG, Li C, Grant MH. Berberine as a therapy for type 2 diabetes and its complications: From mechanism of action to clinical studies 1. Biochemistry\& Cell Biology .2014;1-8.

7.Zhang X, An L, Shao Z. Adverse reactions and treatment of Coptis chinensis and its products, Traditional Chinese Medicine. 2003;1:14-714.

8.Chen W, Miao YQ, Fan DJ, Yang SS, Lin X, Meng LK, et al. Bioavailability study of berberine and the enhancing effects of TPGS on intestinal absorption in rats.AAPS PharmSciTech .2011;12(2):705-11.

9.Fratter BD, Servi. New oral delivery system to improve absorption of berberine区likely interaction of cationized chitosan with PG-P pump.Int J Drug Deliv Tec . 2014;5(1):33-42.

10.Ju Y, Qiu W, Jiang X,Liu C. Effect of BBH on pharmacokinetics of digoxin in rats.Chinese Journal of traditional Chinese Medicine.2011;(7):918-921.

11.Dong $\mathrm{N}$, Liu H, Sun L.Determination of dissolution of BAH tablets and evaluation of its dissolution in vitro.Chinese pharmacy.2018;29(17):8-61.

12.Zhou Z, Hao L, Feng H. Comparative study on dissolution of BAH tablets from different manufacturers .Chinese folk medicine.2018;27(11): 26-32.

13.Zhu LY.Pharmacological effects of berbamine.Chinese Tropical Medicine.2005;(01):109-110.

14.Cheng Y, Qi J, Xiong D, Liu J, Qi S, Pan B,et al.Reversal effect of calmodulin antagonist o - (4-ethoxy) butyl berbamine on multidrug resistant breast cancer cell line MCF-7 / ADR . Journal of Chinese Academy 
of Medical Sciences.2006;028(002):164-168.

15.Liu L, Zhang L, Liu X, Song E. Application of verapamil in common ophthalmic diseases.Jilin medicine. 2010;31(005):674-676.

16.Xu L, Zhao X, Xu R,Wu D.Apoptosis of K562 cells induced by calmodulin antagonist berbamine and its mechanism. Zhejiang University. 2003;024(005):261-262.

17. He H, He H.Effect of Berbamine on DNA damage repair and cataract formation in diabetic cataract rats.Chinese Journal of Biochemistry and molecular biology. 1998;014(3):300-303.

18.Ye F, Yue L, Fan G, Meng X ,Lai X.Effect of Berberis bark water extract on diabetic retinopathy in dB / $\mathrm{db}$ diabetic mice (I). Chinese Journal of experimental pharmacology. 2016;(2):82-86.

19.Zhou B, Ye F,Yue L, Meng X, Fan G ,Lai X.Effect of Berberis bark water extract on diabetic retinopathy in $\mathrm{dB} / \mathrm{db}$ diabetic mice (II). Chinese Journal of experimental pharmacology .2016;022(24):116-121.

20.Yue L, Meng X, Zhang Y,Xiang L,Fan G,Lai X.Effects of Berberis bark on expression of KC, VEGF and HIF-1 a in retina of diabetic rats. World science and technology modernization of traditional Chinese medicine.2014;(1):181-186.

21.Yue L, Meng X, Zhang Y,Xiang L,Fan G, Lai XR.Effects of Berberis bark on expression of KC, VEGF and HIF-1 a in retina of diabetic rats. World science and technology modernization of traditional Chinese medicine.2014;(1):181-186.

22.Zhang Y, Meng X,Yue L, Xiang L ,Lai X.Effect of Berberis bark on blood glucose level in diabetic mice .Progress in Modern Biomedicine. 2013;013(19):3619-3622.

23.He, X., Song. Z.J, Jiang.C.P and Zhang.C.F.Absorption Properties of Luteolin and Apigenin in Genkwa Flos Using In Situ Single-Pass Intestinal Perfusion System in the Rat.The American journal of Chinese medicine.45(8): :1745-1759,2017.

24. Huang S, Long X, Yuan F, Chen L, Cai BM, Qiu H.Study on the absorption mechanism of Puerarin by phenol red method and modified gravimetric method in rats. Journal of Guangdong Pharmaceutical University.2012;28(006):603-607 .

25.Zhang Y, Takao K, Abe C, Sasak K, Ochiai K ,Matsui T.Intestinal Absorption of Prenylated Isoflavones, Glyceollins, in Sprague-Dawley Rats. Journal of Agricultural and Food Chemistry.2020.

26.Ma L, Yang X. Absorption of berberine and coptidine in human Caco-2 cell monolayer model. Chinese Journal of traditional Chinese medicine. 2007;032(023):2523-2527.

27.Li W, Yang X, Zhang J,Fu C,You Y, Lin Y, et al.Effect of Radix Aconiti Lateralis Preparata combined with Glycyrrhiza on intestinal absorption characteristics of aconite alkaloids in rats. Chinese Journal of 
28.Deng S, Lin J, Lin L, Xiao F, Zhang P.In vivo intestinal absorption kinetis of Morinda in rats. Chinese Journal of experimental prescriptions.2015;021(4):89-93.

29.Gao H, Wang M, Sun D, Sun S,Sun C, Liu J ,et al.Evaluation of the cytotoxicity and intestinal absorption of a self-emulsifying drug delivery system containing sodium taurocholate.European journal of pharmaceutical sciences.2017;106:212-219.

30.McInerney MP, Pan Y, Volitakis I, Bush Al, Short JL ,Nicolazzo JA. The Effects of Clioquinol on Pglycoprotein Expression and Biometal Distribution in the Mouse Brain Microvasculature.Journal of pharmaceutical sciences.2019;108(7) : 2247 - 2255.

31.Pan GY, Wang GJ, Liu XD,Fawcett JP ,Xie YY.The involvement of P-glycoprotein in berberine absorption.Pharmacology \& toxicology.2002; 91(4):193-7.

\section{Figures}

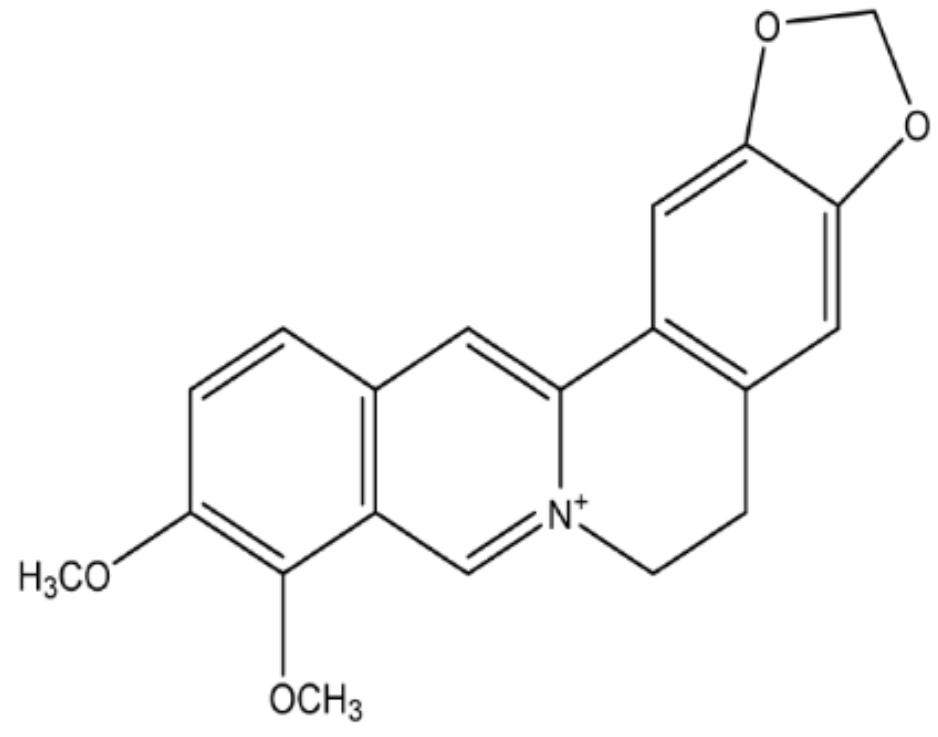

Berberine<smiles>COc1cc2c(cc1Oc1c(OC)c(OC)cc3c1C(Cc1ccc(O)c(Oc4ccc(CC5c6ccccc6CCN5C)cc4)c1)N(C)CC3)C(C)N(C)CC2</smiles>

Berbamie

\section{Figure 1}

Chemical structural formulas of berberine and berbamine 


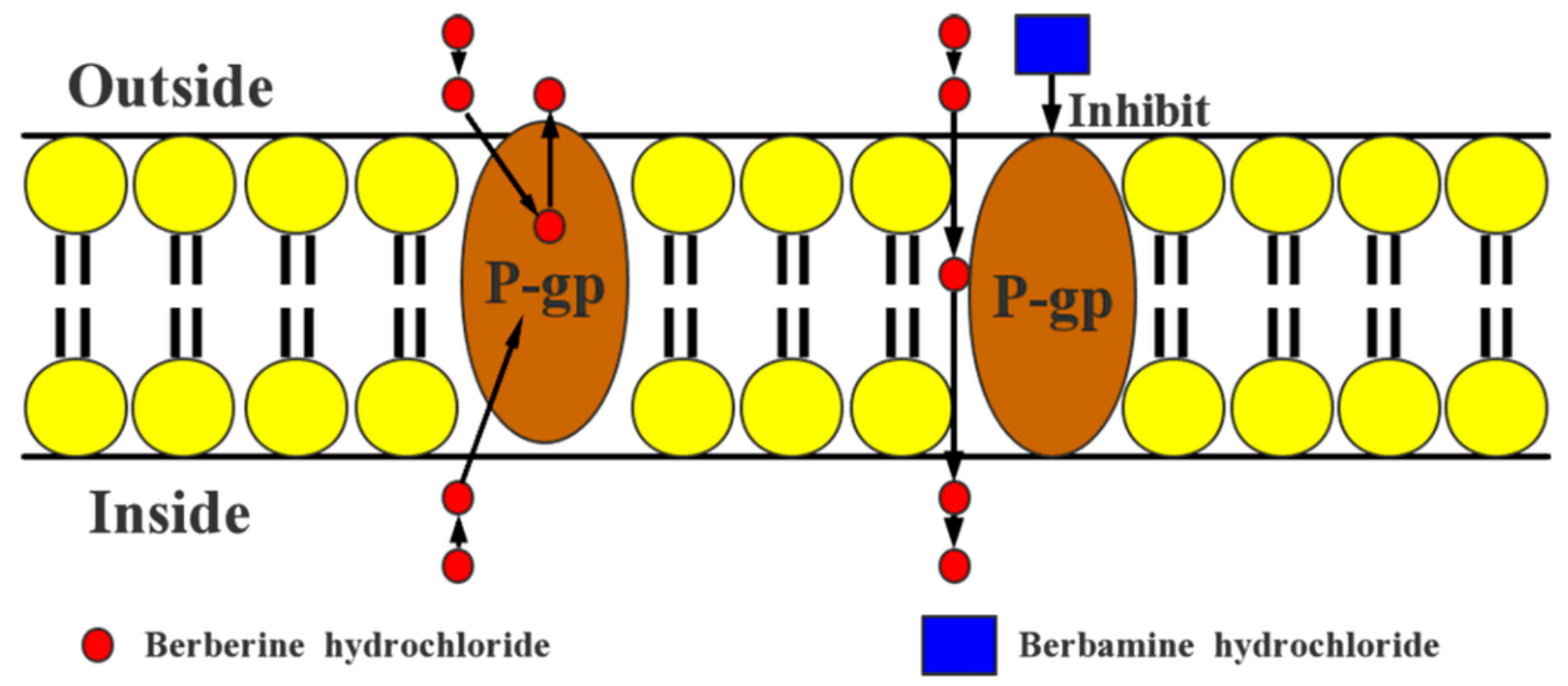

Figure 2

P-gp is P-glycoprotein. On the left side of the figure, berberine will be discharged from the cell by P-gp. On the right side of the figure, when $\mathrm{BBH}$ and $\mathrm{BAH}$ are combined, $\mathrm{BBH}$ The inhibitory effect of amine on the expression of P-gp protein, berberine will enter the cell smoothly.
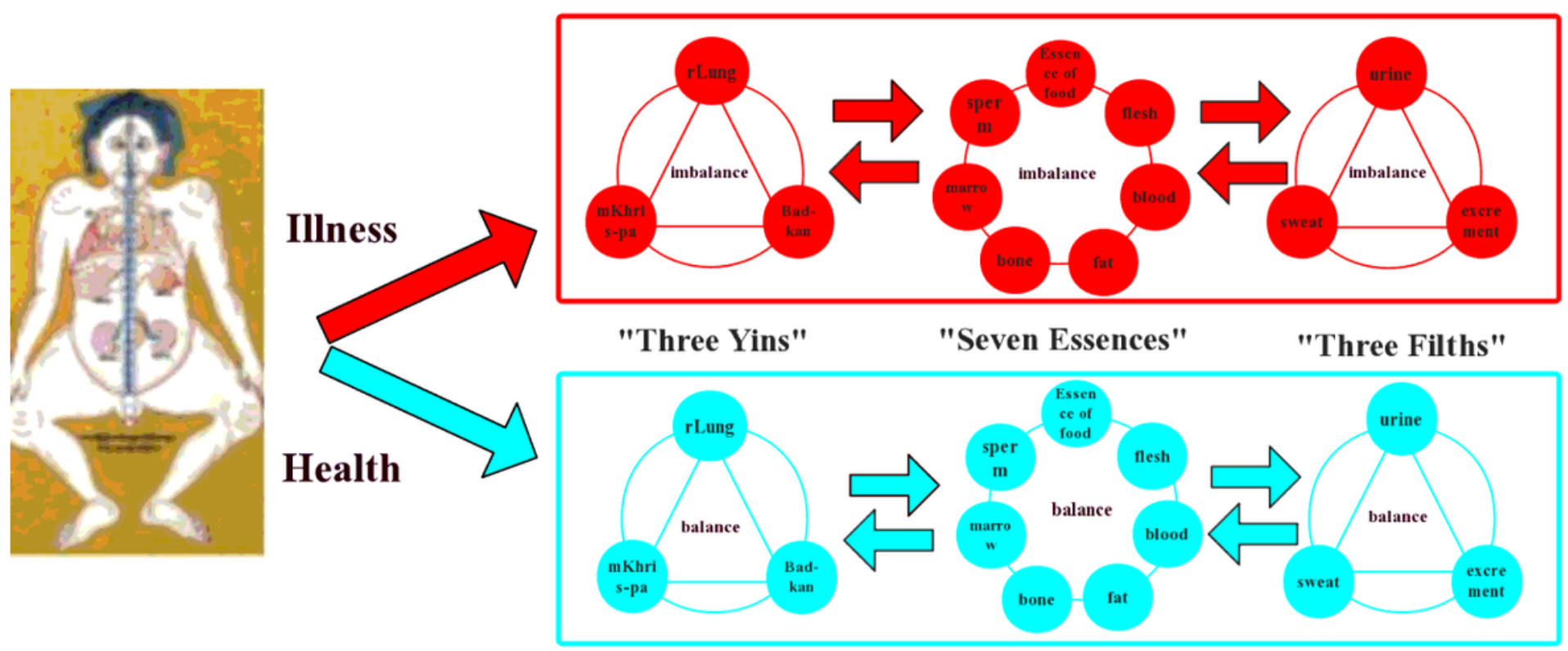

Figure 3

The balance and imbalance of the "three causes", "seven essences" and "three impurities" in Tibetan medicine 


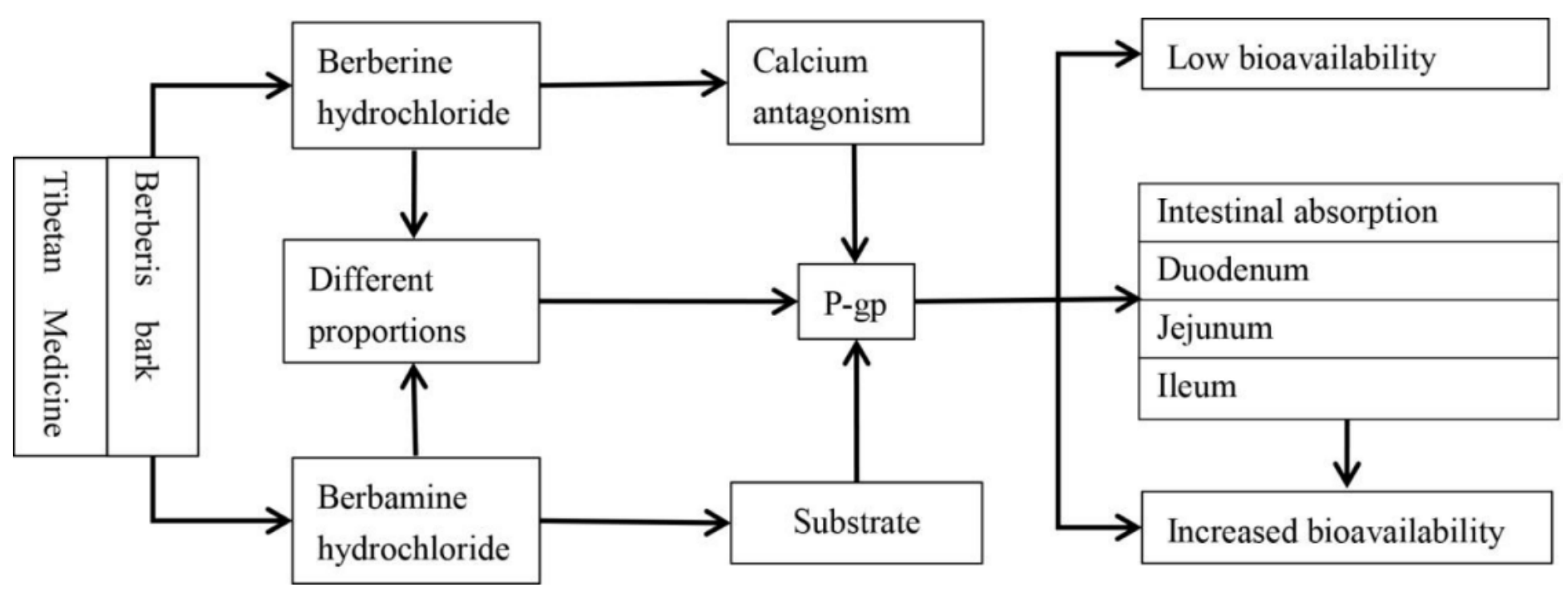

Figure 4

Experimental technical roadmap of the effect of $\mathrm{BAH}$ on the absorption of $\mathrm{BBH}$

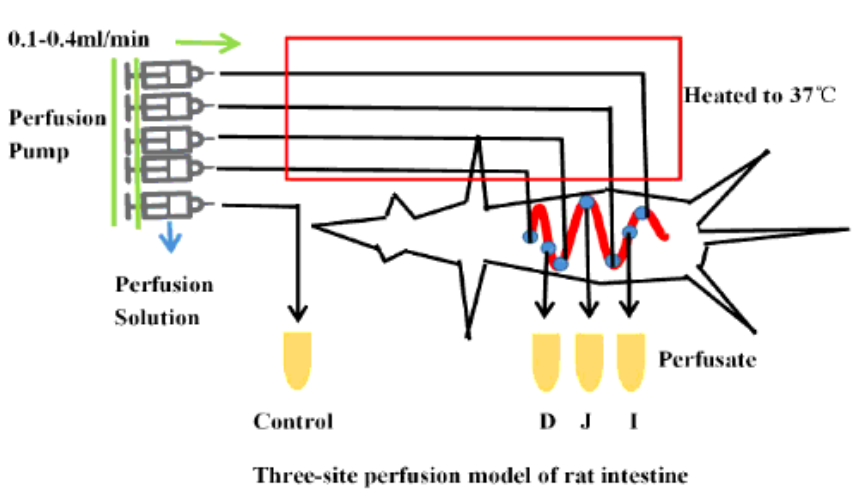

$\mathbf{A}$

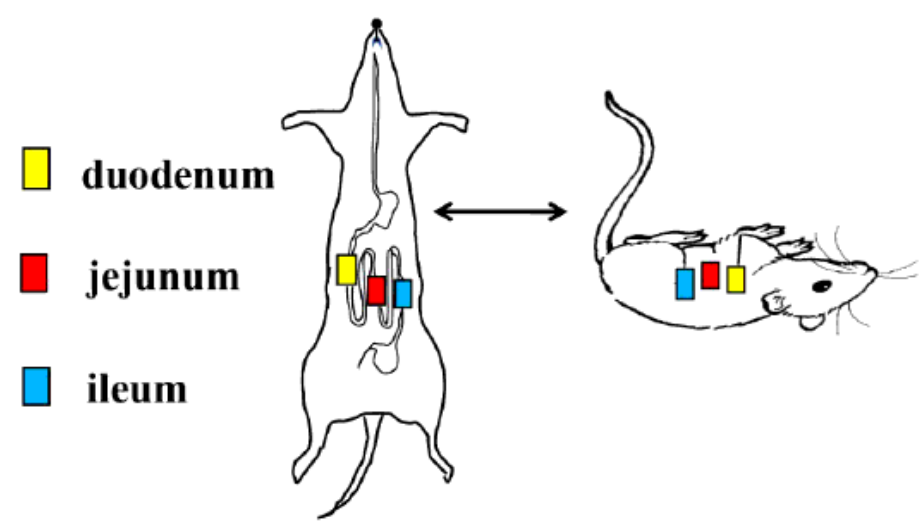

B

\section{Figure 5}

A: Experimental diagram of unidirectional intestinal perfusion in rats. The perfusion rate of intestinal perfusate is $0.35 \mathrm{ml} / \mathrm{min}$. After perfusion of $30 \mathrm{~min}$, the perfusate EP tube and collecting fluid EP tube of known quality were quickly changed in each interval $15 \mathrm{~min}$. The perfusate was collected at the exit $(\mathrm{D}, \mathrm{J}, \mathrm{I})$ until 105 minutes, and the experiment was stopped, that is, the samples were sampled at $45,60,75,90$ and 105 min respectively after timing. 5B: three locations of one-way intestinal perfusion in this experiment. Yellow, red and blue represent the solution of the drug absorbed in duodenum, jejunum and ileum, corresponding to $D, J$ and I of $5 A$, respectively. 

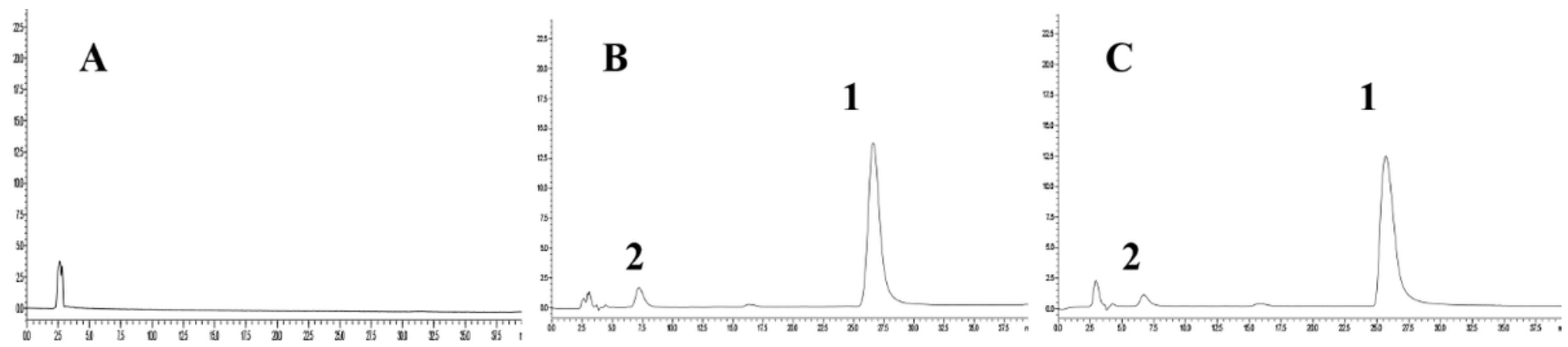

$\mathrm{t} / \mathrm{min}$

Figure 6

A: Blank KR solution with $1 \%$ dimethyl sulfoxide; $6 \mathrm{~B}$ : Blank KR solution with $1 \%$ dimethyl sulfoxide as the reference substance of the test reagent; $6 \mathrm{C}$ : Blank with $1 \%$ dimethyl sulfoxide as the test reagent after perfusion KR solution; 1: $\mathrm{BBH}$; 2: $\mathrm{BAH}$ 


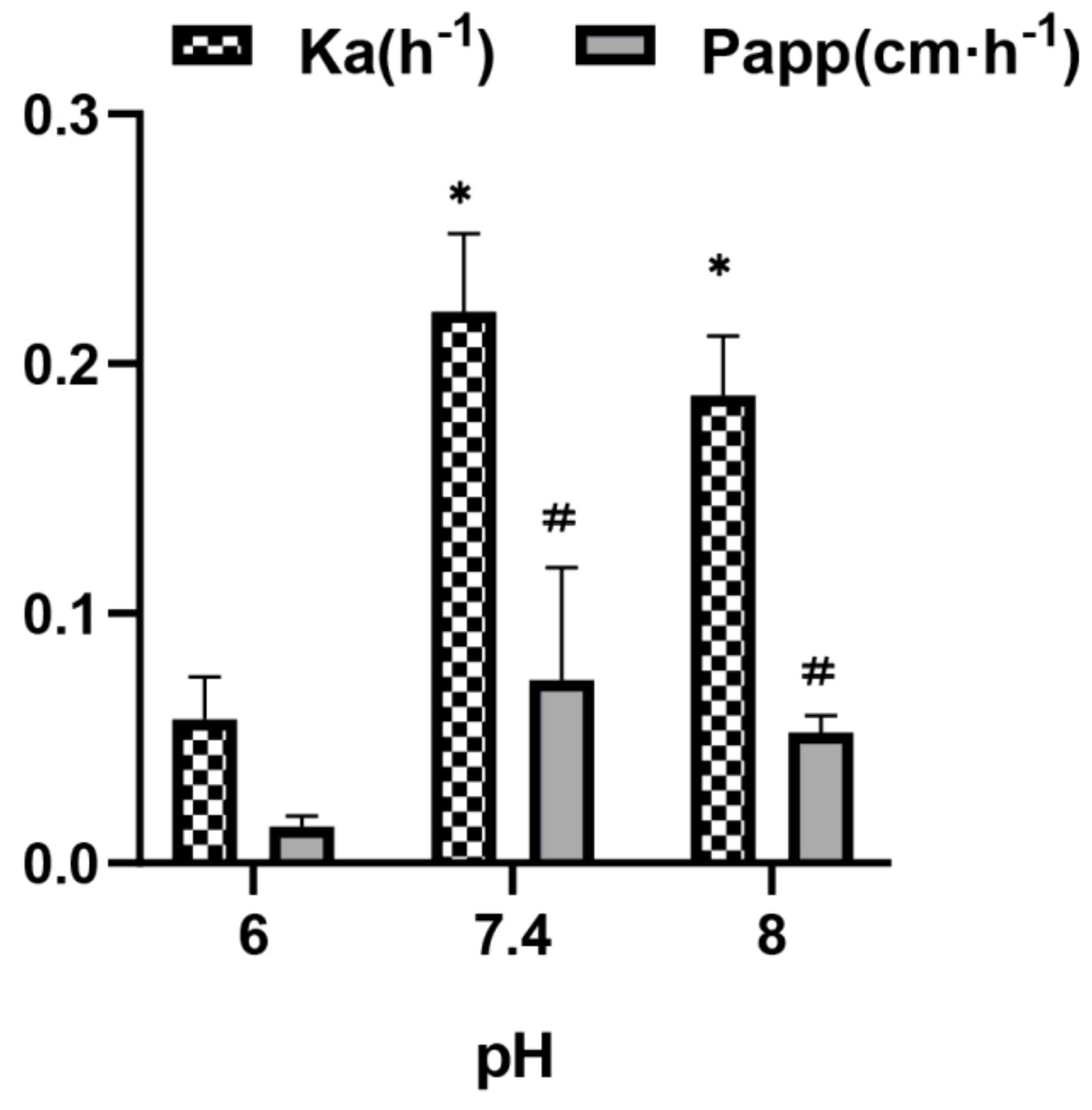

Figure 7

Bar chart of the effect of perfusate of different $\mathrm{pH}$ on the absorption of whole bowel segment of the ratio of $\mathrm{BBH}$ and $\mathrm{BAH}$. The data are expressed as the average \pm standard deviation $(n=6)$. Ka and Papp are perfused at $\mathrm{pH} 7.4$ There is a significant difference between the solution and the $\mathrm{pH} 6$ ratio ${ }^{*} \mathrm{P}<0.05 ; \mathrm{Ka}$, Papp has a significant difference between the $\mathrm{pH} 8$ perfusion solution and the $\mathrm{pH} 6$ ratio $\# \mathrm{P}<0.05$. 


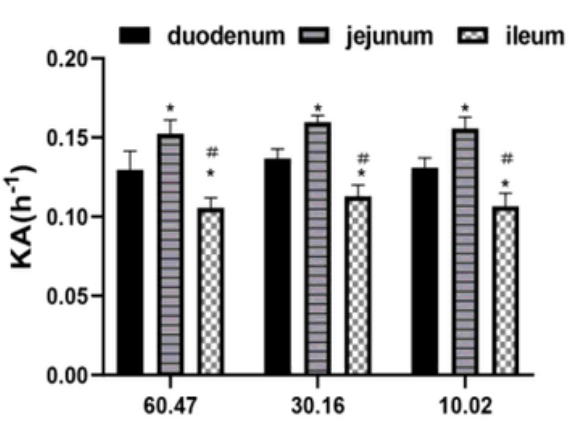

Mass concentration of berberine hydrochloride $\left(\mu \mathrm{g} \cdot \mathrm{ml}^{-1}\right)$

A

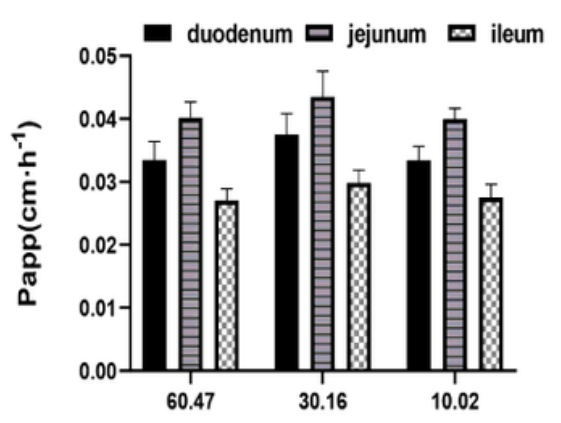

Mass concentration of berberine hydrochloride $\left(\mu \mathrm{g} \cdot \mathrm{ml}^{-1}\right)$

B

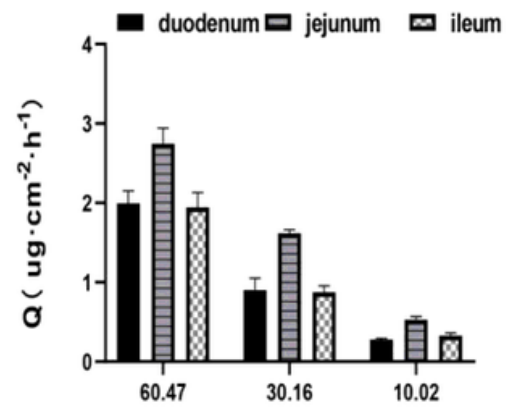

Mass concentration of berberine hydrochloride $\left(\mu \mathrm{g} \cdot \mathrm{ml}^{-1}\right)$

C

Figure 8

Absorption parameters of different mass concentrations of $\mathrm{BBH}$ in each intestinal segment, $8 \mathrm{~A}$ rate constant (Ka), 8B effective permeability coefficient (Papp), 8C cumulative absorption per unit area per hour $(Q)$, the data is average \pm standard deviation $(n=6)$ indicates that the Ka of the duodenum at the same concentration is significantly different from the Ka of the jejunum and ileum * $\mathrm{P}<0.05$; the Ka of the same concentration of the jejunum and the Ka of the ileum also contains a significant difference $\# \mathrm{P}<0.05$.

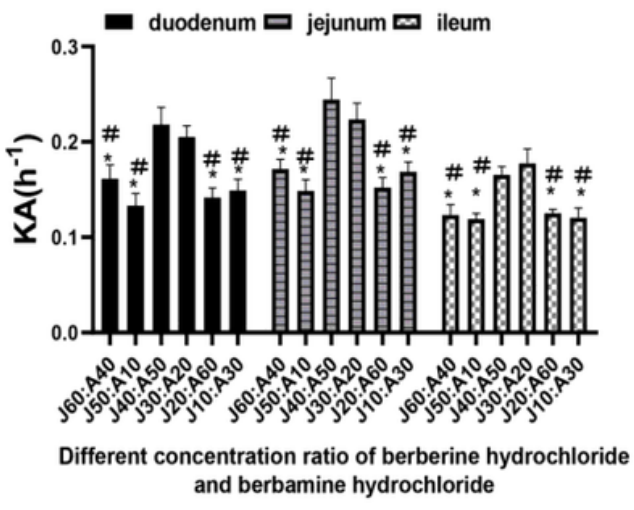

A

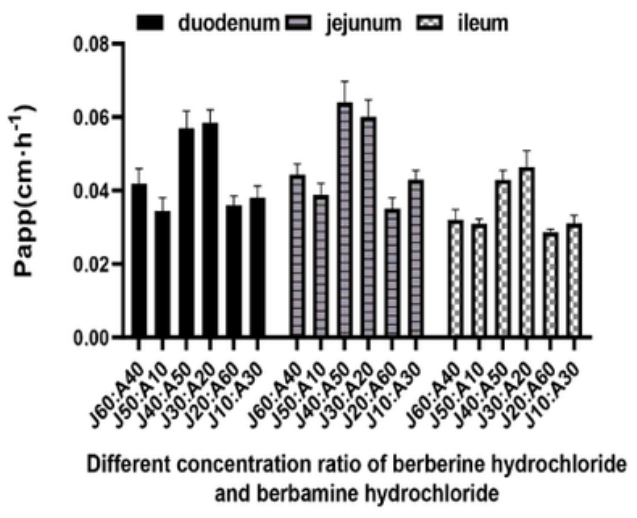

B

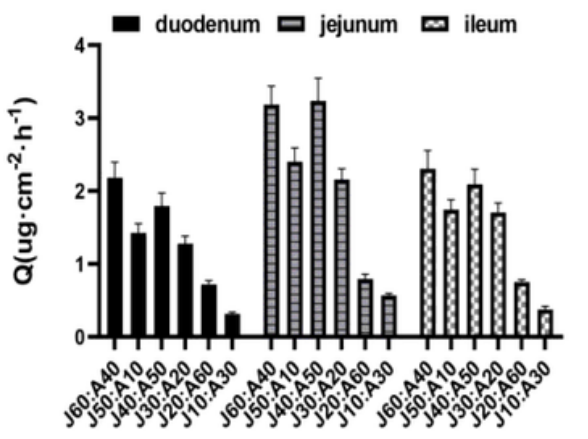

Different concentration ratio of berberine hydrochloride and berbamine hydrochloride

C

\section{Figure 9}

Absorption parameters of different concentrations of $\mathrm{BBH}$ and $\mathrm{BAH}$ in each intestinal segment,9A rate constant (Ka), 9B effective permeability coefficient (Papp), 9C cumulative absorption per unit area per hour (Q).When the $\mathrm{BBH}$ and $\mathrm{BAH}$ ratio group had a mass concentration ratio of $\mathrm{B} 40$ :A50 and B30:A20,the absorption of $\mathrm{BBH}$ were better than that in other matching groups. The data are expressed as mean \pm standard deviation $(n=6)$,Compared with B40:A50, there were significant differences between Ka and other proportions of the same intestinal segment * $P<0.05$; compared with B30:A20, there were significant differences between $\mathrm{Ka}$ and other proportions of the same intestinal segment\#P<0.05. 


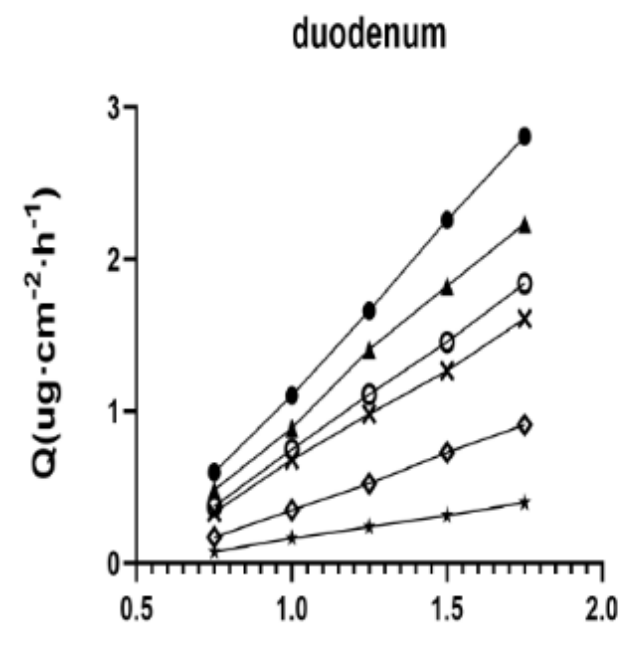

$\mathbf{A}$

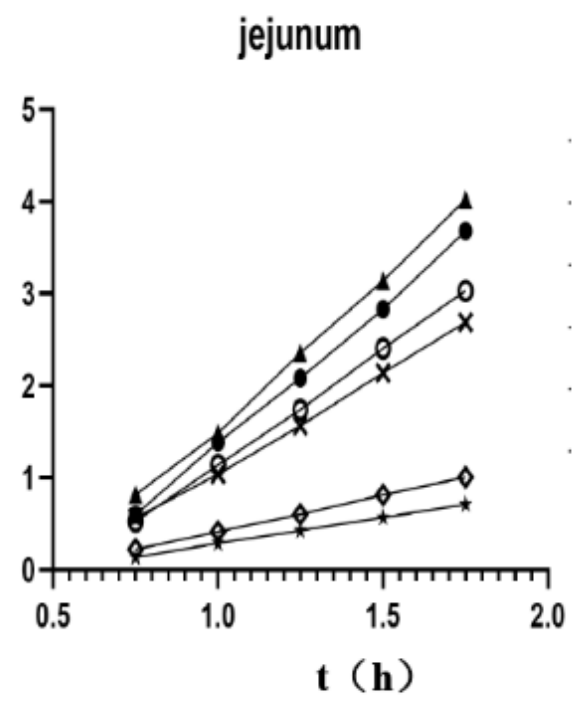

B ileum

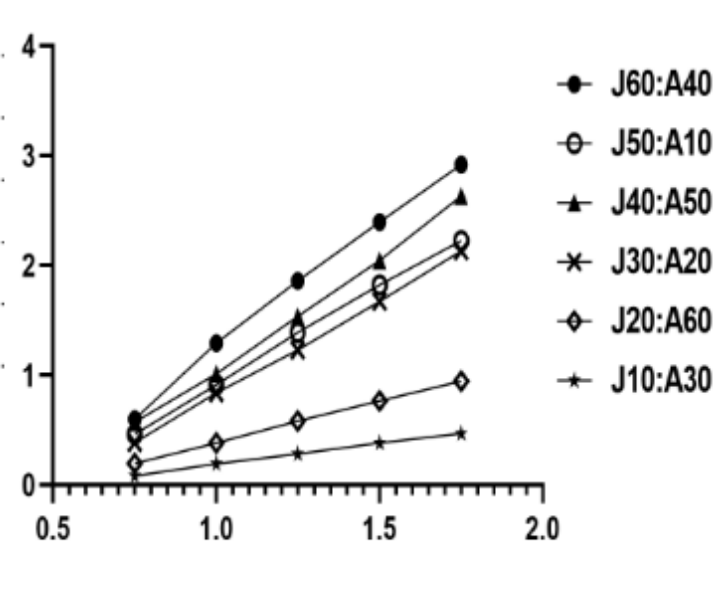

C

Figure 10

Q-t curves of different ratios of $\mathrm{BBH}$ and $\mathrm{BAH}$ absorbed in each intestinal segment, 10A duodenum, 10B jejunum, 10C ileum. 